\title{
SAM domain-dependent activity of PfTKL3, an essential tyrosine kinase-like kinase of the human malaria parasite Plasmodium falciparum
}

\author{
Abdirahman Abdi • Sylvain Eschenlauer • \\ Luc Reininger $\cdot$ Christian Doerig
}

Received: 28 January 2010/Revised: 10 March 2010/Accepted: 22 April 2010/Published online: 27 June 2010

(C) The Author(s) 2010. This article is published with open access at Springerlink.com

\begin{abstract}
Over the last decade, several protein kinases inhibitors have reached the market for cancer chemotherapy. The kinomes of pathogens represent potentially attractive targets in infectious diseases. The functions of the majority of protein kinases of Plasmodium falciparum, the parasitic protist responsible for the most virulent form of human malaria, remain unknown. Here we present a thorough characterisation of PfTKL3 (PF13_0258), an enzyme that belongs to the tyrosine kinase-like kinase (TKL) group. We demonstrate by reverse genetics that PfTKL3 is essential for asexual parasite proliferation in human erythrocytes. PfTKL3 is expressed in both asexual and gametocytes stages, and in the latter the protein colocalises with cytoskeleton microtubules. Recombinant PfTKL3 displays in vitro autophosphorylation activity and is able to phosphorylate exogenous substrates, and both activities are dramatically dependent on the presence of an $\mathrm{N}$-terminal "sterile $\alpha$-motif" domain. This study identifies PfTKL3 as a validated drug target amenable to highthroughput screening.
\end{abstract}

Electronic supplementary material The online version of this article (doi:10.1007/s00018-010-0434-3) contains supplementary material, which is available to authorized users.

A. Abdi - S. Eschenlauer - L. Reininger - C. Doerig Inserm U609, Wellcome Trust Centre for Molecular Parasitology, Glasgow Biomedical Research Centre, 120 University Place, Glasgow, Scotland G12 8TA, UK

L. Reininger · C. Doerig ( $\square)$

Inserm-EPFL Joint Laboratory, Global Health Institute, Ecole Polytechnique Fédérale de Lausanne (EPFL), GHI-SV-EPFL, Station 19, 1015 Lausanne, Switzerland e-mail: christian.doerig@epfl.ch
Keywords Malaria - Protein kinase - Tyrosine kinaselike kinase - SAM domain - MORN motif - Knock-out . Plasmodium falciparum

\section{Introduction}

Malaria is a major public health problem affecting approximately $40 \%$ of the world population [1]. The vast majority of fatal cases are caused by Plasmodium falciparum, one of the five species of malaria parasites that infect humans. The number of deaths due to malaria doubled in the 2 decades preceding the millennium, the primary reason being the spread of parasite resistance to antimalarial drugs, especially chloroquine [2]. Artemisinin combination therapy (ACT) helped reverse this trend, but reduced artemisinin sensitivity was recently reported in southeast Asia [3, 4]. Therefore, development of antimalarials with novel modes of action is an urgent priority.

Protein phosphorylation is recognised as a major mechanism by which virtually every activity of a eukaryotic cell is regulated. Eukaryotic protein kinases (ePKs) are distributed in seven major groups (CK1, AGC, CamK, CGMC, TK, TKL and STE) on the basis of phylogenetic analysis of catalytic domains; the enzymes that do not belong to any of these groups are classified as "OPK" ("other protein kinases") [5]. Based on (1) the success of protein kinase inhibitors in the treatment of various cancers [6] and the prospect of the use of such molecules in microbial diseases [7], and (2) the documented divergences between mammalian and Plasmodium protein kinases (PKs) (reviewed in [8]), the kinome of the parasite has been proposed as an attractive target for pharmacological intervention [9]. The $\approx 85$ enzymes that constitute the $P$. falciparum kinome include several "orphan" kinases 
that have no orthologues in other systems and cluster neither within the seven major ePK groups nor with ePKs in the OPK group [10]. In contrast, Plasmodium "semiorphan" kinases clearly do belong to one of the ePK groups or specific OPK families, but orthology to individual enzymes within these groups/families in other organisms cannot be assigned.

Plasmodium falciparum members of the CK1, CMGC, AGC or CamK groups, as well as plasmodial PKs related to OPKs such as the NIMA-related kinases, have all been the subject of functional investigations (reviewed in [8]). In contrast, the Plasmodium TKLs (Tyrosine kinase-like) have been largely understudied. TKL amino acid sequences are related to those of tyrosine kinases (TKs), a group that is not represented in the parasite's kinome. Some TKL families, such as RAFs and MLKs, are MAP3K (the core module of MAPK pathways consists of a cascade of three kinases, MAP3K [MEKK], MAP2K [MEK] and MAPK). Others like IRAK and RIPK members are involved in immune response signalling, while others such as LIM kinase are regulators of the cytoskeleton. The plasmodial kinome was reported to contain five TKL homologues [10], but the only published report on a TKL concerns the identification of one such kinase (PlasmoDB identifier PF11_0220) as secreted to the extracellular medium [11].

Our interest in plasmodial MAPK pathways prompted us to engage in the characterisation of the $P$. falciparum TKL group, as by analogy with other systems (see above) they represent potential MAP3K candidates. Here, we present a refined phylogeny of the $P$. falciparum TKL group that reduces the number of PfTKLs from 5 to 4 , and we proceed to a detailed characterisation of one of these four enzymes, PfTKL3. We demonstrate that an accessory "Sterile $\alpha$-Motif" (SAM) domain present on the PfTKL3 polypeptide mediates oligomerisation and is crucial for kinase activity in vitro. We provide evidence that PfTKL3 is essential for completion of the erythrocytic asexual cycle, thus validating the enzyme as a potential target for antimalarial intervention.

\section{Materials and methods}

\section{Bioinformatics}

Sequences alignment was performed using T-Coffee (http://www.ebi.ac.uk/Tools/t-coffee/); when necessary the aligned sequences were edited manually. Shading of residues was performed using GeneDoc and the bootstrap neighbour-joining phylogenetic tree generated with the MEGA software 4 [12].
Parasite culture

Asexual parasites of the P. falciparum 3D7 clone were grown as described [13] and used as recipients in all transfection experiments. Gametocytes were prepared according to the protocol of Carter et al. [14].

Plasmid construction

\section{PfTKL3 KDWT}

A 1,155-bp DNA fragment [nucleotides 4,213-5,367 of the predicted coding sequence of PfTKL3 (PF13_0258)] was amplified by PCR from a $P$. falciparum asexual cDNA library (strain IT04), using primers 560 and 541 (see Supplemental Table 1) containing BamH1 and Sal1 sites, respectively. The amplified fragment was cloned into pET28a between the BamH1 and Sal1 restriction sites.

\section{PfTKL3 dead mutant-pET28a}

Mutations of the specific residues $(\mathrm{K} \rightarrow \mathrm{M}$ and $\mathrm{D} \rightarrow \mathrm{N})$ were introduced following the method of [15] by two rounds of PCR with specific primers $(\mathrm{K} \rightarrow \mathrm{M}$ : primers 688 and $689, \mathrm{D} \rightarrow \mathrm{N}$ : primers 652 and 653 ) containing the mutations (see Supplemental Table 1).

\section{PfTKL3 Sam-KD and SAM domain mutagenesis}

The region spanning nucleotides $3,922-5,367$ of the PfTKL3 coding sequence, which includes the region coding for the SAM and the kinase domains, was PCRamplified from a $P$. falciparum asexual cDNA library using primers 690 and 541, containing BamH1 and Sal1 restriction sites, respectively (see Supplemental Table 1). The insert was cloned into pET28a plasmid using the same procedure as the kinase domain. Two residues, H35 and V54 (numbering is from the start of the cloned SAM domain) within the SAM domain, were mutated to Glu by two rounds of PCR using primers (first round: V54E: primers 733 and 734, H35E: primers 779 and 780) containing the appropriate mutation and second round: primers 690 and 541. The mutants were cloned into pET28a plasmid described for the wild type.

pCAM-BSD-PfTKL3

A 561-bp DNA fragment (nucleotides $4,540-5,100$ of the PfTKL3 ORF) was amplified by PCR from $P$. falciparum genomic DNA using primers 501 and 549 (with BamH1 and Not 1 restriction sites, respectively) and was inserted into pCAM-BSD [16]. 
pCAM-BSD-PfTKL3-TAG

The pCAM-BSD-TAG vectors were generated by introducing the sequence coding for GFP or double HA (dHA), and the $3^{\prime}$ UTR of the $P$. berghei dhfr-ts gene into the multiple cloning site of pCAM-BSD (Fig. 3a). The $3^{\prime}$ end of the PfTKL3 coding region (621 bp omitting the stop codon) was amplified by PCR from genomic DNA, using primers 577 and 578 with Pst 1 or Bam $\mathrm{H} 1$ restriction sites, which allowed insertion of the amplified product into pCAM-BSD-TAG.

\section{Cloning of the SAM domain}

The sequence encoding the SAM domain $(3,922-4,131$ of the ORF) was PCR amplified using primers 690 and 737 and cloned into pET28a, pGEX4T3, or pMAL-c2E as described above. A stop codon was included in the reverse primer sequence.

Expression and purification of recombinant proteins

All expression vectors were transformed into Rosetta2 (DE3)pLysS cells (Stratagene). pET28-transformed cells were grown in LB media containing $10 \mu \mathrm{g} / \mathrm{ml}$ of kanamycin and $34 \mu \mathrm{g} / \mathrm{ml}$ of chloramphenicol, and expression was induced with $1 \mathrm{mMIPTG}$ at $20^{\circ} \mathrm{C}$ overnight. For the plasmids based on the pGEX4T3 and pMAL-c2E vectors, $100 \mu \mathrm{g}$ ampicillin was used instead of kanamycin, and induction was with $0.2 \mathrm{mM}$ IPTG. Purification of $\mathrm{His}_{6}$-tagged proteins was performed on Ni-NTA agarose beads (Qiagen), while the GST- and MBP-tagged proteins were purified on glutathione(Sigma) and amylose- (NEB) agarose beads.

\section{cDNA preparation and RT-PCR}

Total RNA was extracted from parasite pellets using TRIzol lysis solution (Invitrogen). DNase treatment of RNA samples prior to RT-PCR was performed by incubation at $37^{\circ} \mathrm{C}$ for $30 \mathrm{~min}$ with RQ1 RNase-free DNase (Promega). The DNase was inactivated by incubation at $65^{\circ} \mathrm{C}$ for $10 \mathrm{~min}$. To generate cDNA, RETROscript ${ }^{\circledR}$ Kit (Ambion) was used; $2 \mu \mathrm{g}$ of RNA was used with random decamers in a PCR with or without reverse transcriptase following the manufacturer's protocol. The cDNA was then used in nested PCR using primers 560 and 541 (first round) and 501 and 541 (second round). The PfTKL2 kinase domain (loading control) was amplified from the cDNA in a single step PCR using primers 935 and 505.

\section{Western blotting}

Parasite extracts: Parasite pellets obtained by saponin lysis from synchronised asexual cultures and gametocytes cultures were incubated in lysis buffer $[50 \mathrm{mM}$ Tris, pH 8.0, $300 \mathrm{mM} \mathrm{NaCl}, 0.1 \mathrm{mM}$ EDTA, 0.5\% Triton, $1 \times$ protease inhibitors cocktail (Roche)] for $30 \mathrm{~min}$ on ice, briefly sonicated and spun at $14,000 \mathrm{rpm}$ for $10 \mathrm{~min}$ at $4^{\circ} \mathrm{C} ; 10 \mu \mathrm{g}$ from the supernatants was boiled in Laemmli sample buffer, run on a $8 \%$ SDS-PAGE gel and transferred to nitrocellulose (transfer buffer: $25 \mathrm{mM}$ Tris, pH8.3, $192 \mathrm{mM}$ glycine, $20 \%$ methanol and $0.025 \%$ SDS). Membranes were blocked in 5\% skimmed milk (Bio-Rad) and PBS containing $0.25 \%$ Tween 20 for $1 \mathrm{~h}$ at room temperature. Washes were performed using PBS containing $0.25 \%$ Tween 20 . Rat anti-HA mAb,clone 3F10, Roche (1:500), mouse anti-GFP, mAb Roche (1:500) and chicken anti-Rat-HRP, Abcam $(1: 2,500)$ and rabbit anti-mouseHRP $(1: 5,000)$ were used. Detection was performed by chemoluminescence using the ECL system (Perkin Elmer). In the case of anti-GFP, 1 volume of SuperSignal West Femto Substrate (Pierce Biotechnology) and 2 volumes of Perkin-Elmer detection reagent were mixed for detection of PfTKL3-GFP signal. Rabbit anti-PfGR $(1: 15,000)$ and anti$P$. falciparum thioredoxin peroxidase $1(1: 4,000)$ antibodies (gift from Prof. Sylke Muller) were used as loading control.

Recombinant proteins: Transfer of the recombinant protein was performed using the semi-dry cell (Bio-Rad). Rabbit Anti-GST (Sigma) (1:10,000), rabbit anti-MBP (NEB), (1:10,000) and rabbit anti-His 6 (Santa Cruz) $(1: 1,000)$ primary antibodies were used. Anti-rabbit IgGHRP (Sigma) $(1: 10,000)$ secondary antibody was used.

\section{Parasite transfection}

Transfections were carried out by electroporation of ring stage 3D7 parasites with $50-100 \mu \mathrm{g}$ of plasmid DNA, according to [16]. Blasticidin (Calbiochem) was added to a final concentration of $2.5 \mu \mathrm{g} / \mathrm{ml} 48 \mathrm{~h}$ post transfection.

\section{Genotype characterisation}

\section{PCR}

For the detection of integration of the pCAM-BSDPfTKL3 plasmid into genomic DNA, the following primers were used (see Fig. 2a for numbering and see Supplemental Table 1 for sequences): 560 (forward) and 803 (reverse) diagnostic for wild-type locus, 167 (forward) and 168 (reverse) for episome, 560 and 168 for $5^{\prime}$ integration, and 167 and 803 for $3^{\prime}$ integration. For detection of integration of the pCAM-BSD-PfTKL3-GFP/HA plasmid, the following primers were used (see Fig. 3a for numbering and see Supplemental Table 1 for the sequences): 560 and 803 for wild type, 167 and 803 for $3^{\prime}$ integration, 560 and 806/639 (reverse for GFP and HA-tags respectively) for $5^{\prime}$ 
integration, and 167 and 806 (or 168 in the case of HA) for episome.

\section{Southern blotting}

Genomic DNA was extracted from parasite pellets prepared from the transfectant lines using QIAamp ${ }^{\circledR}$ DNA mini kit (Qiagen). The DNA was digested with $N$ sil, separated on a $0.8 \%$ agarose gel and transferred to a Hybond $\mathrm{N}$ membrane (Amersham). Probing with DNA probes was carried out using the Amersham AlkPhos direct labelling and detection kit. Briefly, $100 \mathrm{ng}$ of a DNA fragment (either the fragments inserted into the knock-out or tagging plasmids) was labelled and hybridised with the membrane overnight at $55^{\circ} \mathrm{C}$. Labelling of the probe, washes of the membrane, and detection were performed as described in the manufacturer's protocol. The membrane was stripped with $0.1 \%$ SDS (in $\left.\mathrm{dH}_{2} \mathrm{O}\right)$ at $55^{\circ} \mathrm{C}$ for an hour and reprobed with a probe derived from the BSD coding region.

\section{Microscopy}

Live parasites expressing GFP-tagged PfTKL3 and parasites fixed with $4 \%$ paraformaldehyde were used. Images of live parasites were captured directly using a Delta vision deconvolution fluorescence microscope (X100 Olympus IX-70 objective). For fixed parasites, images were obtained after counterstaining (1) with mouse anti-Pfs16 antibody (1:50) (a kind gift from Pietro Alano) or mouse antichicken-brain-tubulin (1:250) (clone DM1A, purchased from Sigma) and (2) with 4,6-diamidino-2-phenylindole, and a TRITC-conjugated goat anti-mouse IgG1 secondary antibody (1:250) (Southern Biotech, Birmingham, AL).

\section{Kinase assays}

Kinase assays were performed in a $30-\mu 1$ reaction mixture containing the recombinant (either wild-type or mutant) protein, $5 \mu \mathrm{g}$ of substrate (myelin basic protein, histone $\mathrm{H} 1, \alpha$-casein and $\beta$-casein), $20 \mathrm{mM} \mathrm{MgCl} 2,2 \mathrm{mM} \mathrm{MnCl}$, $10 \mu \mathrm{M}$ ATP (containing $0.075 \mathrm{MBq}[\gamma-32 \mathrm{P}]$ ), $10 \mathrm{mM} \mathrm{NaF}$ and $10 \mathrm{mM} \beta$-glycerophosphate in $20 \mathrm{mM}$ Tris ( $\mathrm{pH} 7.5$ ). The mixture was incubated for $30 \mathrm{~min}$ at $30^{\circ} \mathrm{C}$, and the reaction was stopped by adding Laemmli buffer and heating at $100^{\circ} \mathrm{C}$ for $3 \mathrm{~min}$. The samples were resolved by SDS-PAGE gel, and the gel was stained with Coomassie blue, dried and incubated with X-ray film (Kodak) and subjected to autoradiography.

SAM domain expression and pull down assays

The SAM domain coding sequence was cloned into pET28a, pGEX4T3 and pMAL-c2E and expressed in
Rosetta2(DE3)pLysS E. coli. pGEX4T3 and pMAL-c2E were also transformed into the same cells and induced for expression of GST and MBP alone. The recombinant proteins were purified using Ni-NTA agarose beads, glutathione beads or amylose beads, respectively. For pulldown assays, purified MBP-SAM was incubated while shaking with purified GST-SAM for $1 \mathrm{~h}$ at $4^{\circ} \mathrm{C}$. As a control, MBP-SAM was similarly incubated with GST or lysis buffer (50 mM Tris, $100 \mathrm{mM} \mathrm{NaCl}, 0.1 \mathrm{mM}$ EDTA, pH 7.4). Glutathione beads pre-washed with the lysis buffer were added to the mixture and incubated for another $1 \mathrm{~h}$ at $4^{\circ} \mathrm{C}$. Similarly, purified GST-SAM was incubated as above with MBP-SAM, MBP alone or lysis buffer. Amylose beads were added to the mixture and incubated as above. The beads were centrifuged at $1,500 \mathrm{rpm}$ for $2 \mathrm{~min}$, washed three times (washing buffer: $50 \mathrm{mM}$ Tris, $300 \mathrm{mM}$ $\mathrm{NaCl}, 0.1 \mathrm{mM}$ EDTA, $\mathrm{pH}$ 8.0) and eluted with wash buffer containing either $15-\mathrm{mM}$ reduced glutathione or $12-\mathrm{mM}$ maltose. Eluted proteins were boiled in Laemmli buffer, resolved on SDS-PAGE gel, transferred to nitrocellulose paper and probed the paper with both anti-GST (rabbit, Sigma) and anti-MBP (rabbit, NEB) antibodies.

\section{Results}

\section{Bioinformatic analysis}

Phylogenetic analysis of the $P$. falciparum kinome [10] identified five sequences clustering with the human TKL group, with PlasmoDB identifiers PFB0520w, PF11_0220, PF13_0258, PFF1145c, and PF14_0320; the latter was a relative outlier, branching out close to the base of the TKL cluster. A more refined analysis of kinase domains including several additional human sequences of the TKL group (Fig. 1a) now leads us to conclude that PF14_0320 cannot be classified as a TKL, but confirms that PFB0520w, PF11_0220, PF13_0258, PFF1145c (thereafter called PfTKL1, PfTKL2, PfTKL3 and PfTKL4, respectively) do indeed belong to the TKL group. PfTKL1 and PfTKL3 cluster closely together and have similar accessory functional domains within their $\mathrm{N}$-terminal extension (see below). The present study focuses on PfTKL3 (PF13_0258).

The pftkl3 gene is predicted to comprise a single exon located on chromosome 13, encoding a 1807-residue protein. The kinase catalytic domain (Pfam entry PF00536, E-value $1.4^{-43}$ ) is located in the $\mathrm{C}$-terminal region (residues 1407-1789). The long N-terminal extension comprises the following domains, starting from the N-terminus (Fig. 1b): (1) a putative PEXEL motif (Plasmodium Export Element, RXLX[EQD]) mediating trafficking to the host erythrocyte (residues 25-RNLDD-29); (2) a potential 14-3-3 mode II 


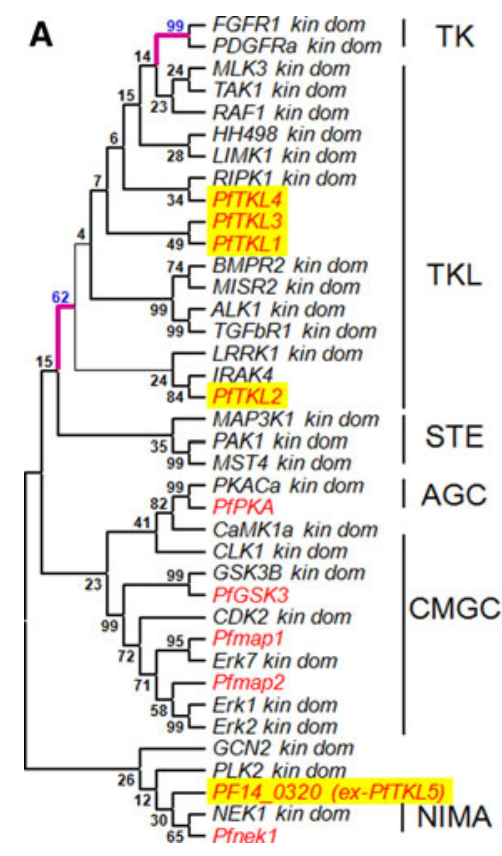

Fig. 1 Phylogeny and domain organisation of PfTKL3. a Phylogenetic tree of the kinase domain sequences from representatives of the human (black) and P. falciparum (red) kinome families. The five

binding motif (RX[YF]Xp[TS]xP) (55-RRFKTEP-61); (3) two tandem putative MORN motifs (Membrane Occupation and Recognition Nexus) motif (residues 475-497 and 498519); Pfam entry PF02493, E-value 98); and (4) a putative SAM domain (residues 1,311-1,372; Pfam entry PF00532, E-value $1.8^{-05}$ ). MORN motifs are involved in membrane interactions, while SAM domains are protein-protein interaction mediators. The E-value for the MORN motif is low, but sequence alignment with proteins that contain multiple MORN motifs, such as Arabidopsis thaliana phosphatidylinositol kinase, confirms the presence of a MORN motif in PfTKL3 and orthologs (Supplemental Figure S1A).

The pftkl3 gene is conserved in all the Plasmodium and Cryptosporidium species (C. parvum and C. hominis) for which genome sequence data are available. A homolog is also present in Neospora caninum and Toxoplasma gondii (gene identifiers; NCLIV_008190 and TGME49_053860 respectively). In $T$. gondii, the $\mathrm{N}$-terminal extension is very short in comparison to the other apicomplexan homologues, and lacks the MORN and SAM motifs within the extension. The N-terminal extensions of Plasmodium and Cryptosporidium sequences share no similarity outside the MORN and SAM motifs, with the exception of a highly conserved stretch of unknown function located between the MORN and SAM domains (Fig. 1b displays a schematic representation of the orthologs, and Supplemental Fig. S1B an alignment of the PEXEL, MORN and SAM motifs, the conserved regions of unknown function and the kinase sequences identified as $P$. falciparum TKLs in Ref. [10] are highlighted in yellow. b Schematic representation of PfTKL3 homologs in Apicomplexa

catalytic domain). The potential 14-3-3 binding motif is found only in the Plasmodium and Neospora sequences.

PfTKL3 plays a crucial role in erythrocytic schizogony

To investigate whether the enzyme plays an essential role in erythrocytic schizogony, we attempted to disrupt the pftkl3 locus using a single cross-over gene disruption strategy that we previously used successfully for several $P$. falciparum protein kinases [17-19]. A construct containing the central part of the kinase domain coding region and a blasticidin-resistance cassette was introduced into 3D7 parasites. Single cross-over recombination is expected to disrupt the gene and yield two truncated, non-functional copies (Fig. 2a). Two independent transfections were carried out, and blasticidin-resistant populations were readily obtained. Integration was monitored by PCR (Fig. 2b) and Southern blot (Fig. 2c). Even after prolonged cultivation (6 months), no integration was detected in the transgenic lines. However, increasing PCR sensitivity by using high concentrations of template DNA and 35 PCR cycles allowed us in a few instances to detect a very weak band diagnostic of integration event (data not shown), suggesting that pftkl3 gene disruption is possible, but that the parasites in which integration has occurred could not overgrow the parasites that retain a wild-type pftkl3 locus. This indicates that the modification is either lethal or causes a severe growth rate defect. 
Fig. 2 Targeted gene disruption of $p f t k l 3$. a Gene disruption strategy. The restriction enzyme (Nsi1) sites used for Southern blot analysis and the sizes of the expected fragments are shown. The arrows (dark bold) indicate the location of primers used in PCR analysis. BSD is a blasticidinresistance marker. b PCR analysis. The amplicons are diagnostic for: lane 1, episome; lane $2,3^{\prime}$ boundary of integrated plasmid; lane $3,5^{\prime}$ boundary of integrated plasmid; lane 4, wildtype locus. The numbers 560 , 167, 168 and 803 represent the primers used (sequences in Supplemental Table 1). c Southern blot analysis. Genomic DNA from untransfected (U) and pCAM-BSD-PfTKL3 transfected (T) 3D7 parasites was digested with $N s i 1$ and probed with PfTKL3 (left panel) and BSD (right panel) probes

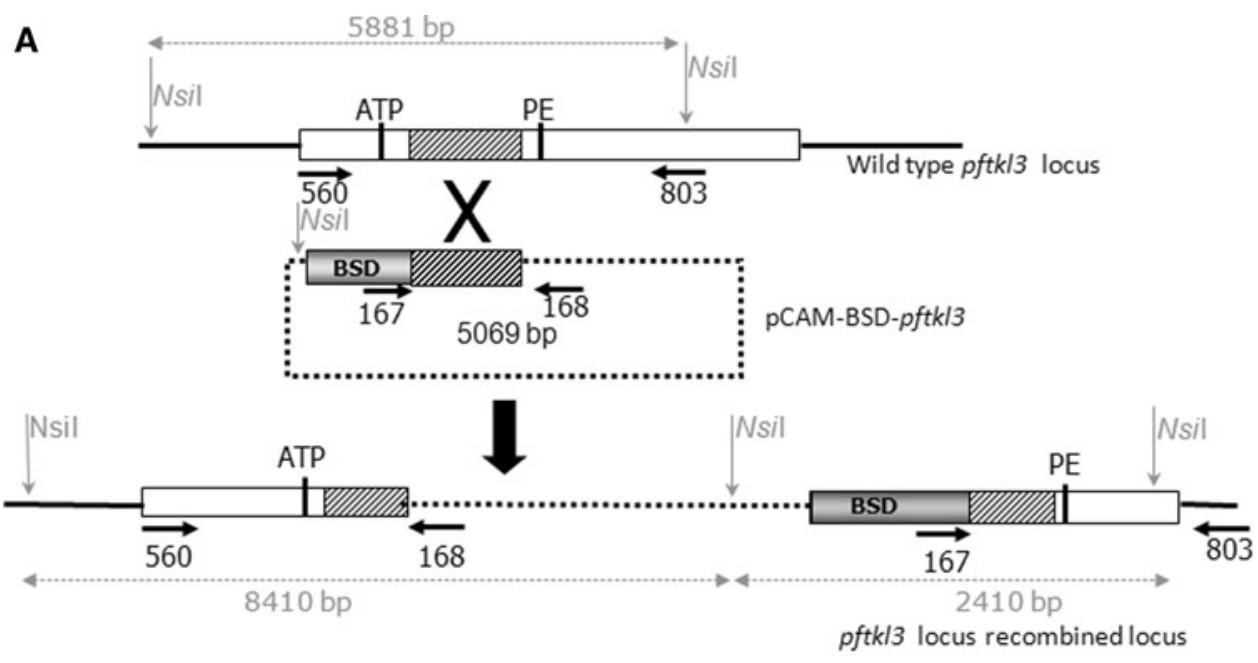

B untransfected transfected
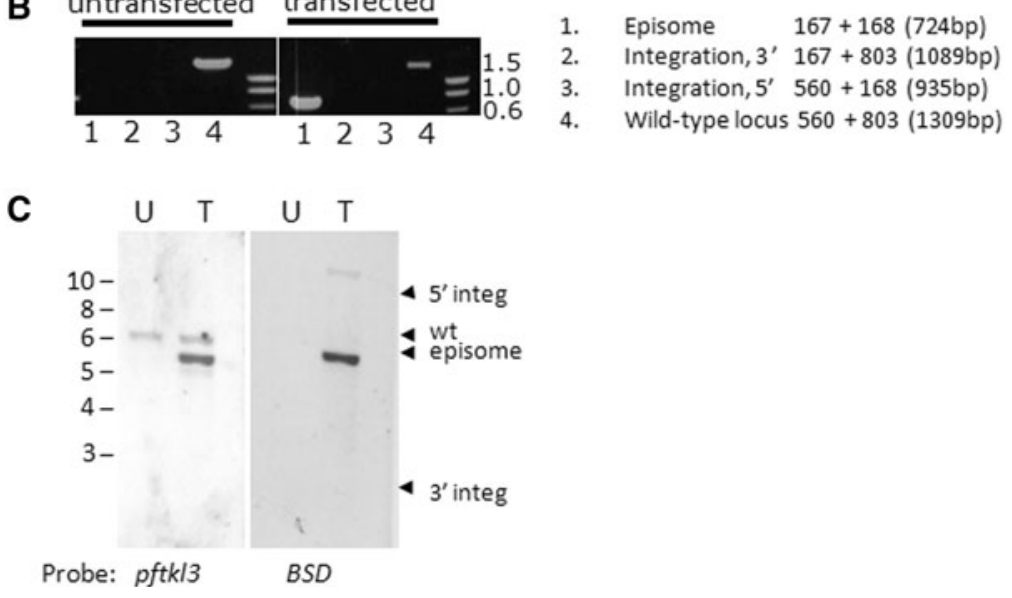

To verify that we could obtain parasite lines with a modified pftkl3 locus if the modification does not cause loss of function, we generated parasite lines expressing the endogenous PfTKL3 protein fused to green fluorescent protein (GFP) or a double hemaglutinin epitope (dHA; Fig. 3a). PCR analyses showed that in transgenic populations integration had taken place by 8 weeks post transfection and that the wild-type locus was lost in both the GFP and dHA-tagged lines (Fig. 3b, c). The PCR result was further confirmed by Southern blot analysis in the case of the GFP-tagged line (Fig. 3d). These results show that the $p f t k l 3$ locus is accessible to homologous recombination.

Expression of epitope-tagged PfTKL3 in asexual culture was first confirmed by using HA- and GFP-specific antibodies in Western blot analyses. Total protein extracts from saponin lysed parasite pellets were prepared from the dHAand GFP-tagged lines, each providing a negative control for the other. Western blot analysis using a rat anti-HA mAb consistently detected 250-, 130- and 95-kDa bands in protein extracts prepared from the HA-tagged line, the latter two bands presumably being $\mathrm{C}$-terminal fragments resulting from processing or degradation of HA-tagged PfTKL3 (the predicted size of the PfTKL3 polypeptide is $212 \mathrm{kDa}$ ) (Fig. 3e). In some experiments a band of higher molecular weight $(>250 \mathrm{kDa})$ was also detected (data not shown). An anti-GFP mAb detected a band of a relatively larger size compared to the HA-tagged protein, as expected from the sizes of the tags. No cross-reactivity between extracts was detected for both antibodies, and equal loading was ascertained with an antibody against $P$. falciparum glutathione reductase (PfGR, PlasmoDB identifier PF14_0192).

\section{PfTKL3 expression profile}

Microarray analysis detected PfTKL3 mRNA in all stages of the asexual cycle, as well as in gametocytes and sporozoites [20] (data available in PlasmoDB, www. plasmodb.org). Nested RT-PCR using RNA extracted from rings, trophozoites and schizonts confirmed the presence of PfTKL3 mRNA throughout the asexual cycle, with mRNA abundance peaking at the schizont stage. 
Fig. 3 GFP- and HA-tagging of the $p f t k l 3$ locus. a C-terminal tagging strategy. The location of PCR primers used for genotyping is indicated by numbered arrows. See the text for details. b PCR analysis of the GFP tagged locus: Genomic DNA from untransfected and transfected 3D7 parasites was subjected to PCR analysis using the primers numbered in $\mathbf{a}$. The amplicons are diagnostic for: lane 1, episome; lane 2, wild type; lane 3, 3' boundary of integrated plasmid; lane $4,5^{\prime}$ boundary of integrated plasmid. The numbers 560, 167, 168, 803 and 806 represent the primers used (sequences in

Supplemental Table 1). c PCR analysis of the HA-tagged locus. This was performed as in $\mathbf{b}$, except the DNA was from pCAM-BSD-PfTKL3dHAtransfected parasites, and primer 639 was used instead of primer 806. d Southern blot analysis. Genomic DNA from untransfected (U) and pCAMBSD-PfTKL3GFP-transfected (T) 3D7 parasites was digested with $N s i 1$ and probed with a pftkl3 probe. e Western blot analysis. Protein extracts (10 $\mu \mathrm{g} /$ lane) from dHA-tagged (lane 1) and GFP-tagged (lane 2) lines were separated by SDSPAGE, transferred to nitrocellulose and probed with anti-GFP (left), anti-HA (right) or anti-PfGR (bottom, loading control) antibodies

A

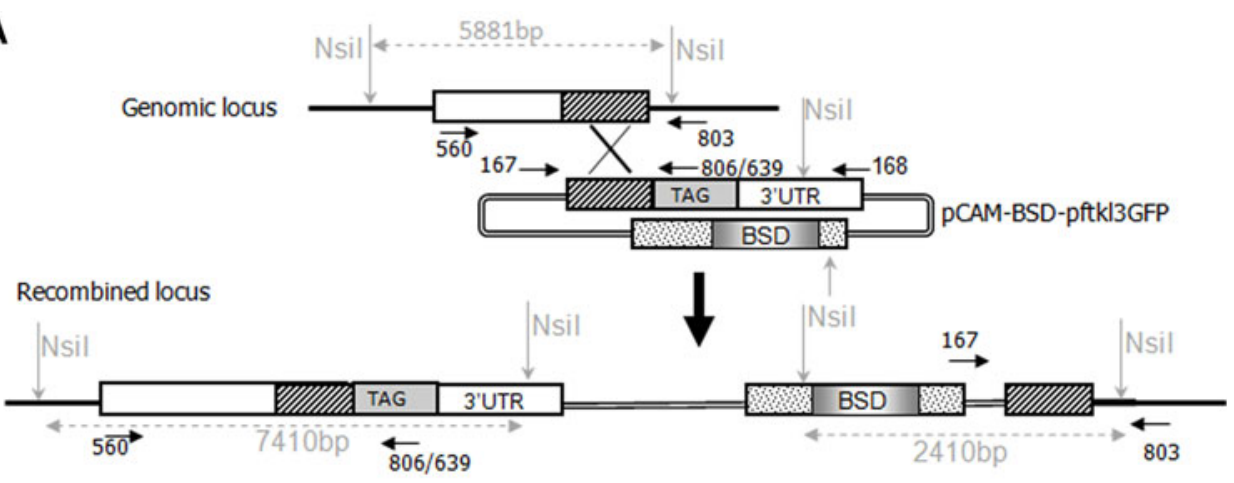

B

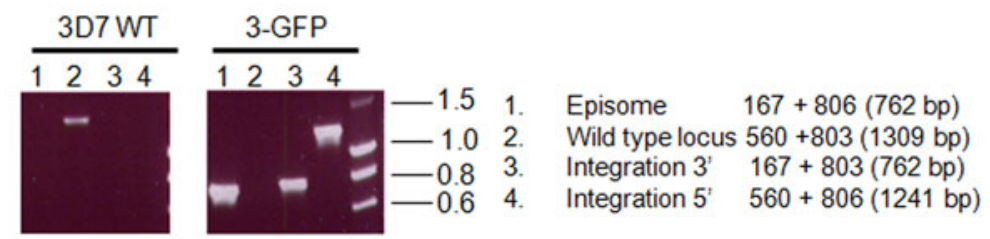

C

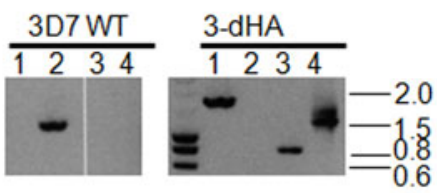

$\begin{array}{lll}\text { 1. } & \text { Episome } & 167+168(1592 \mathrm{bp}) \\ \text { 2. } & \text { Wild type locus } & 560+803(1309 \mathrm{bp}) \\ \text { 3. } & \text { Integration } 3^{\prime} & 167+803(716 \mathrm{bp}) \\ \text { 4. } & \text { Integration } 5^{\prime} & 560+639(1271 \mathrm{bp})\end{array}$

D

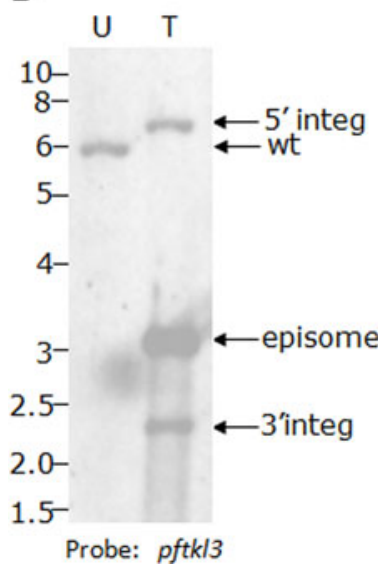

E
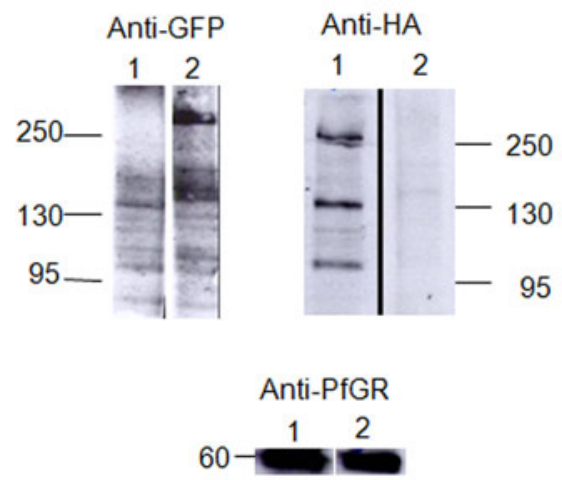

Control reactions without reverse transcriptase did not yield any signal, ruling out genomic DNA contamination. Primers specific for PfTKL2, which is constitutively expressed [11], were used to verify that all samples contained equal amounts of mRNA (Supplemental Fig. S2A).

We used the parasite lines expressing tagged PfTKL3 to monitor expression of the protein. Protein extracts were prepared from rings, trophozoites, schizonts and gametocytes of the PfTKL3-dHA and PfTKL3-GFP lines. Western blot analysis using an anti-HA mAb detected a signal in all extracts from PfTKL3-dHA parasites, while no signal was observed in the control GFP-tagged line. An antibody against $P$. falciparum thioredoxin peroxidase 1 (PfTPx, PF14_0368) was used as a loading control. The PfTKL3/
PfTPx ratio was the highest in schizonts, somewhat lower in trophozoites and very low in rings (Supplemental Fig. S2B).

\section{Fluorescence microscopy}

To gain further insight into the spatial-temporal expression profile of PfTKL3, the PfTKL3-GFP tagged line was analysed microscopically. When live asexual synchronised cultures were observed by fluorescence microscopy, a GFP signal was detected in schizonts and free merozoites (Fig. 4). No signal was detected in rings and trophozoites (not shown). In schizonts, the signal seems to be confined within the parasite (implying the putative PEXEL is not functional) and to localise within structures forming a 


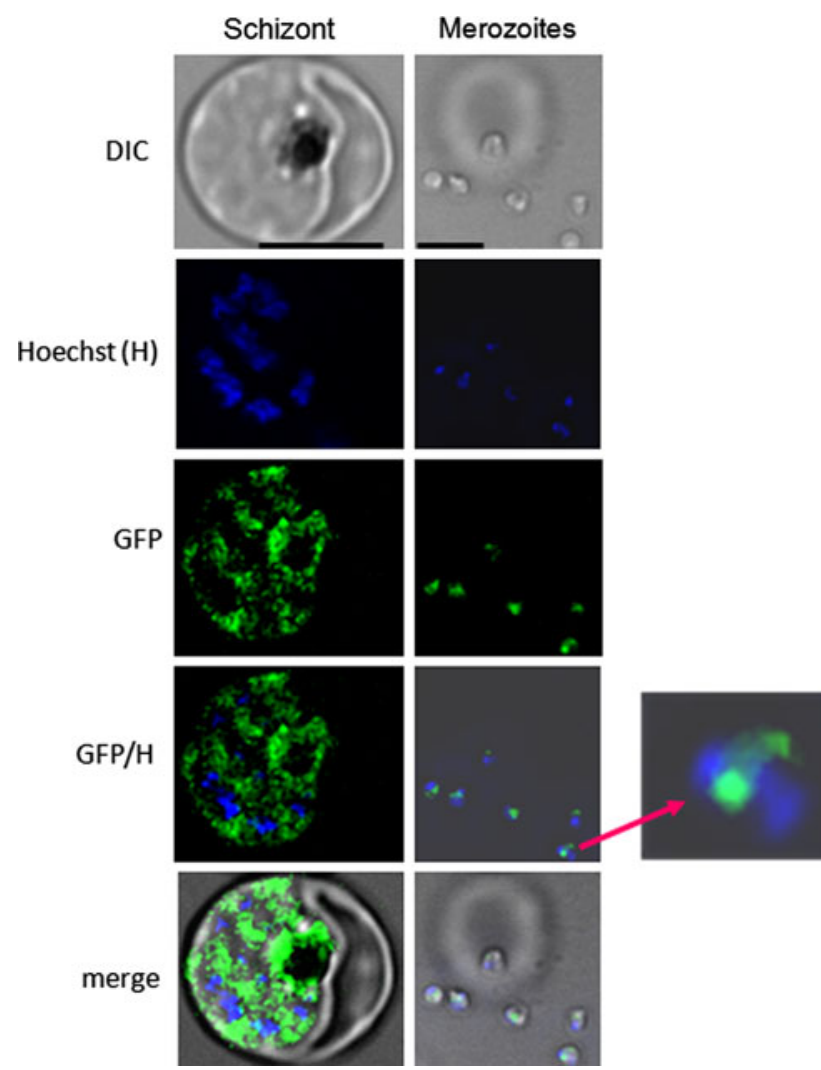

Fig. 4 Visualisation of GFP-tagged PfTKL3 in asexual parasites. Live asexual cultures from the GFP-tagged line were observed by fluorescence microscopy. A signal was detected only in the schizont and free merozoites. A typical image is shown for both these stages. A $4 \times$ relative enlargement of a merozoite is shown to the right. Scale bar: $5 \mu \mathrm{m}$

network that becomes extensive in mid and late schizont stages. Our attempt to assign localisation of the protein to known subcellular structures in the asexual stages was not successful, partly due to the fact that the GFP signal disappears after fixing the cells, thus preventing immunofluorescence analysis. In free merozoites, the protein appears to be localised in an elongated structure with bulbous ends (see the enlarged merozoite in Fig. 4). All stages of gametocyte development gave a GFP signal (Fig. 5). In stage I, the protein appears to be evenly distributed in the cytoplasm (Fig. 5a), whereas in stage II, the signal appears to start localising in discrete areas (Fig. 5b). This becomes more apparent in stage III, where the signal concentrates into a discrete, diamond-shaped area (Fig. 5c, d). In some instances an extension appears to form tubularlooking structures (Fig. 5e, f). In stages IV and V, this becomes less prominent (Fig. $5 \mathrm{~g}, \mathrm{~h}$ ), and the signal is again more evenly distributed, with thread-like structure along the length of one side of the cell. IFA using an antibody against Pfs16 (PFD0310w), a protein that resides within the parasitophorous vacuole membrane, revealed that the GFP signal is confined within the gametocyte (Fig. 6a).
Since the GFP signal accumulates in thread-like structures along one side of the cell, we next investigated if this is associated with the sub-pellicular membrane complex, which has been described as having these characteristics and is thought to anchor the microtubular cytoskeleton, thereby maintaining the shape of gametocytes [21]. We used an antibody against $\alpha$-tubulin to counterstain gametocytes from the PfTKL3-GFP line (this was not possible with asexual parasites, as the GFP signal was lost upon fixation, presumably because of lower amounts of PfTKL3 in asexual parasites than in gametocytes). This showed that the GFP signal co-localises or closely associates with microtubules in the early stages of the gametocytes (Fig. 6b).

\section{Regulation of recombinant PfTKL3 kinase activity}

The sequence encoding the PfTKL3 kinase domain (KD; see Fig. 1b) was amplified by PCR from a cDNA library, expressed as a $\mathrm{His}_{6}$-tagged protein in E. coli, and the recombinant protein was purified on nickel beads. Kinase activity was assessed using non-physiological potential substrates including myelin basic protein (MBP), histone $\mathrm{H} 1$, and $\alpha$ - and $\beta$-casein. Weak activity against MBP and autophosphorylation were observed only when a high concentration of the enzyme was used in the kinase assay (Fig. 7a). In view of published evidence that an accessory SAM domain can positively regulate the activity of KDs in other contexts [22], we produced another recombinant protein that included the SAM domain in addition to the KD (see Fig. 1b). Only a minute amount of this His $_{6}$-tagged SAM-KD protein was recovered (Fig. 7b), presumably because of low solubility (see below). Nevertheless, kinase activity against MBP was considerably higher than that yielded by a similar amount of KD alone, and autophosphorylation was dramatically increased (Fig. 7c). To verify that the observed activity was due to the recombinant SAM-KD rather than to co-purified bacterial contaminants, we repeated the experiment with two "kinase dead" mutant versions of the recombinant proteins. These mutants carry a point mutation abolishing kinase activity (either the change of a conserved Lys in sub-domain II to Met, or the change of a conserved Asp in sub-domain VIB to Asn, see Supplemental Fig. S1B). Reactions conducted with either type of dead mutant did not yield any signal (Fig. 7d). The active wild-type GST$\mathrm{KD}$ phosphorylated the inactive His- $\mathrm{KD}(\mathrm{K} \rightarrow \mathrm{M})$ mutant in a concentration-dependent manner (Fig. 7e), suggesting PfTKL3 autophosphorylation occurs through an intermolecular mechanism.

The effect of $2 \mathrm{mM} \mathrm{Mg}^{2+}, \mathrm{Mn}^{2+}, \mathrm{Ca}^{2+}, \mathrm{Co}^{2+}, \mathrm{Ni}^{2+}$ and $\mathrm{Zn}^{2+}$ ions (chloride salts) on the activity of the SAM-KD form of the enzyme was tested. $\mathrm{Mn}^{2+}$ and $\mathrm{Co}^{2+}$ were found 
Fig. 5 Visualisation of GFPtagged PfTKL3 in gametocytes. A live gametocyte from the PfTKL-GFP line was observed by fluorescence microscopy. Signals were observed throughout gametocyte development. See text for details. Scale bar: $5 \mu \mathrm{m}$

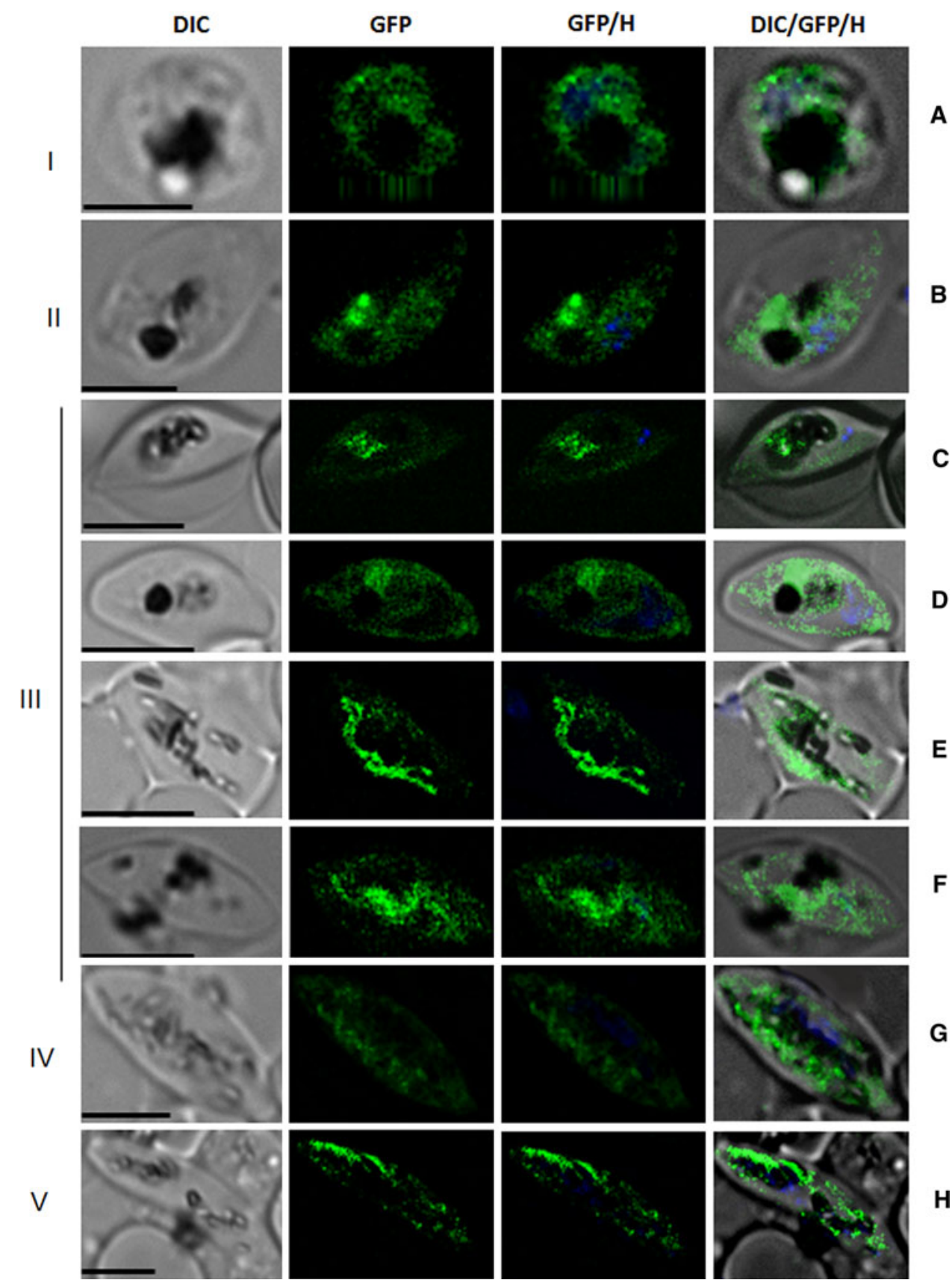

difference in recovery of recombinant KD versus SAM-KD proteins may thus be caused by a lower solubility of the latter. Two residues, Ala61 and Val80, are crucial for polymerisation of the well-characterised ETV6 SAM domain. The structure of ETV6 SAM consists of five helices and has two interacting interfaces: mid-loop (ML) and end-helix (EH), where Ala61 and Val80 are located, respectively. The EH valine is conserved in the PfTKL3 SAM domain (V54), while the ML alanine is substituted by a histidine (H35; Fig. 8b). We mutated these two residues to glutamate, a residue with opposite properties to those of valine (which is non-polar) and histidine (which is SAM domain likewise mediates polymerisation, the 
Fig. 6 Co-localisation studies. a An antibody against the parasitophorous vacuole membrane protein Pfs 16 was used to counterstain PfTKL3GFP expressing gametocytes. b An anti-tubulin antibody (anti-TubA) was used to counterstain PfTKL3-GFP expressing gametocytes. The white arrows indicate where the GFP and TubA signal concentrate in late stages of gametocytes. See text for details. Scale bar: $5 \mu \mathrm{m}$
A
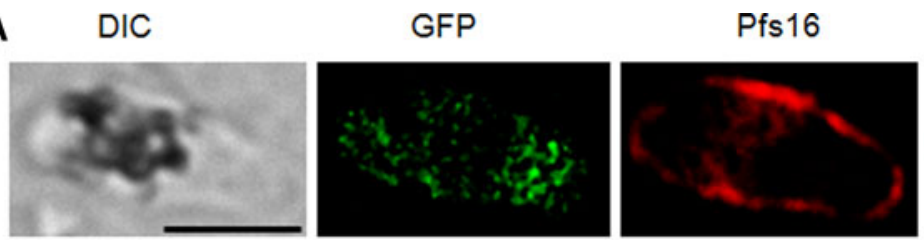

GFP/Pfs16

B

DIC

GFP
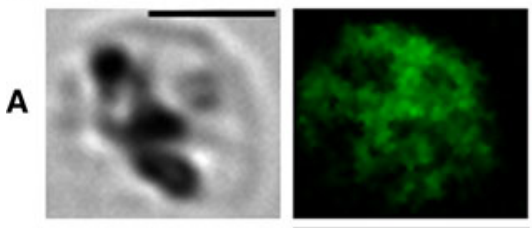

TubA

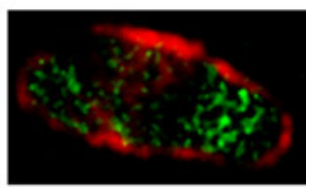

B
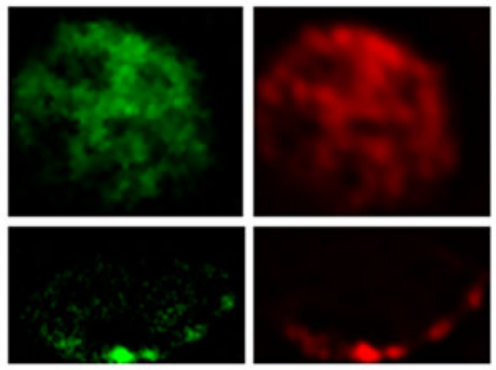

GFP/TubA/DAPI
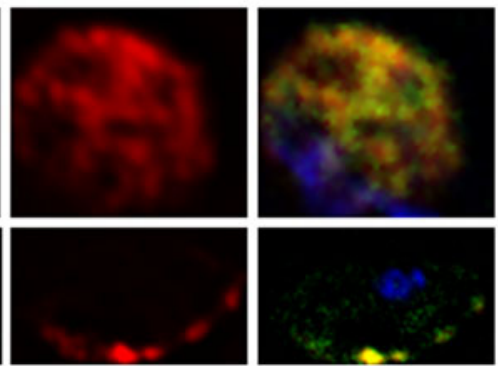

C
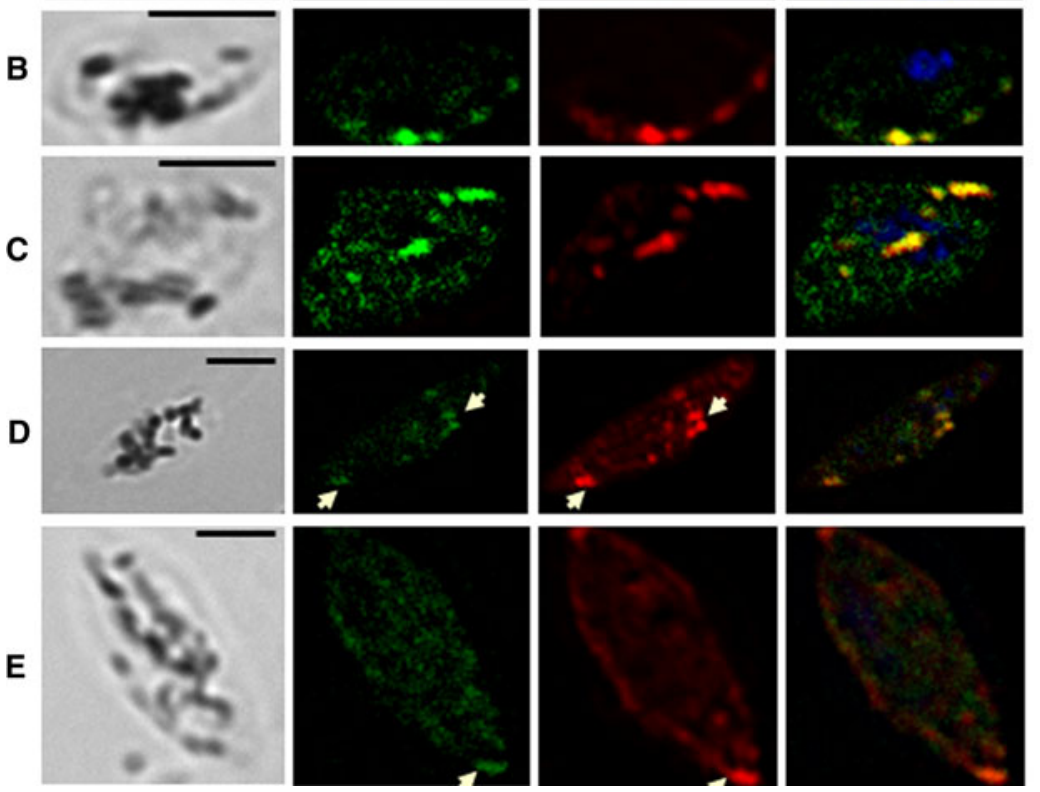

positively charged), in order to disrupt potential interactions mediated by these amino acids, and expressed the mutant SAM-KD as His $_{6}$-tagged recombinant proteins. Both mutations improved the amount of soluble recombinant protein recovered relative to the wild type, as shown by Western blot analysis using a rabbit anti-His 6 antibody (Fig. 8c). This was particularly evident with the V54E mutant, whose yield was sufficient for detection by Coomassie staining (Fig. 8c). This suggests that the conserved Val54 allows the wild-type PfTKL3 SAM domain to form poorly soluble oligo-/polymers and that mutating this residue disrupts the interaction, thereby improving the solubility of the protein.

\section{Self-interaction of the PFTKL3 SAM domain}

We cloned and expressed the sequence encoding the SAM domain (approximately $8 \mathrm{kDa}$ ) as recombinant $\mathrm{His}_{6^{-}}$, GSTand MBP-tagged proteins (Supplemental Fig. 4a). No His $_{6}{ }^{-}$ tag protein was recovered, either in the soluble or in the insoluble fractions, while GST-tagged SAM was largely found in the insoluble fraction (not shown). When the soluble fraction of the GST-tagged form was subjected to batch purification using glutathione agarose beads, only a small fraction of the total protein could be eluted, while most remained in the beads. However, the MBP-tagged form of PfTKL3 SAM domain was readily expressed in a soluble form. Pull-down experiments showed that MBPSAM is able to interact and pull down GST-SAM from solution, and vice versa, while the tags alone used as negative controls were unable to pull down any protein (Fig. 9 and Fig. S4B).

\section{Discussion}

PfTKL3 phylogeny, domain organisation and function in the life cycle

Our phylogenetic analysis (Fig. 1a) supports the original classification of four plasmodial kinases into the TKL group. Among those, PfTKL1 and PfTKL3 share overall 
A

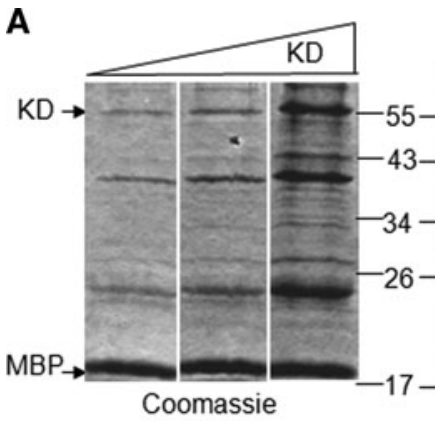

C

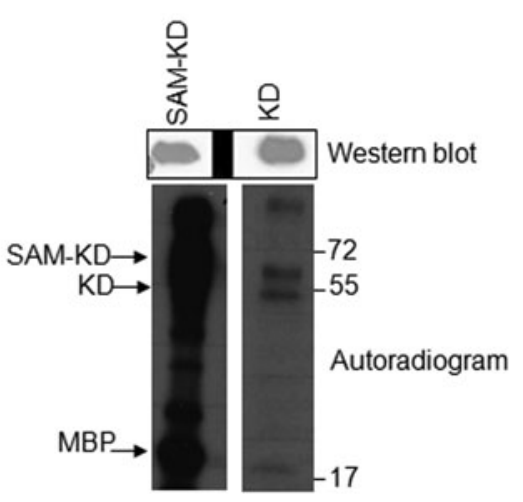

B

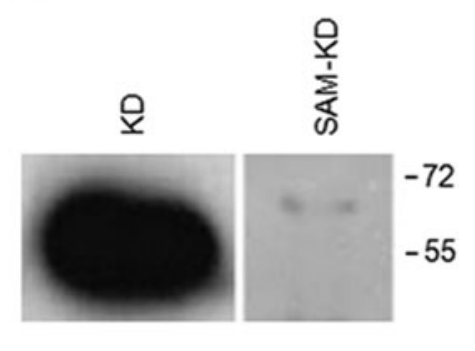

D

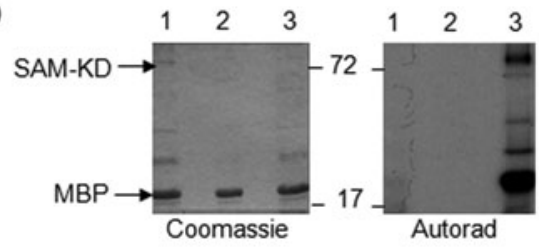

E

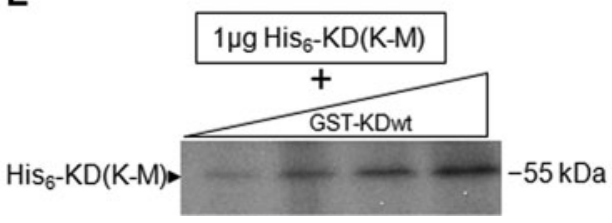

Bottom panel: autoradiogram. d Kinase dead-mutant controls. Kinase assays were performed with the wild-type recombinant SAM-KD PfTKL3 (lane 3), and with the $\mathrm{K} \rightarrow \mathrm{M}$ (lane 1) or $\mathrm{D} \rightarrow \mathrm{N}$ (lane 2) kinase-dead mutants using MBP as substrate. Left panel: Coomassie staining of the gel; right panel: autoradiogram. e Autophosphorylation in trans: $1 \mu \mathrm{g}$ of the catalytically inactive $\mathrm{His}_{6}$-tagged $(\mathrm{K} \rightarrow \mathrm{M})$ mutant kinase domain was used as a substrate in kinase assay using increasing amounts of PfTKL3-KD (twofold serial dilutions from the highest point, which contained $6 \mu \mathrm{g}$ of active wild-type GST-PfTKL3 KD)

PfTKL3 was annotated as "sexual stage-specific protein domain organisation, with MORN and SAM motifs $\mathrm{N}$-terminal to the kinase domain. This organisation is not seen in any mammalian TKLs. This, together with the kinase-domain based phylogenetic tree (Fig. 1a) showing that no human TKL can be identified as a PfTKL1/3 orthologue, concur to label these enzymes as "semiorphans". The Cryptosporidium, N. caninum and T. gondii kinomes include PfTKL3 orhtologues, suggesting a conserved function in apicomplexan biology. PfTKL3 being a "semi-orphan" TKL, it is not possible to predict its cellular function from its sequence. Our reverse genetics data strongly suggest a crucial role for PfTKL 3 in the erythrocytic asexual cycle, at least in the in vitro cultivation system. Elucidation of the cellular process(es) in which the enzyme functions requires addition work; the development of a robust inducible expression system for $P$. falciparum will represent a major breakthrough for functional analysis of essential protein kinases. kise null for proteins containing this 60-70-residue alpha helixrich domain cannot undergo sexual differentiation, hence the name "sterile $\alpha$-motif". Although all SAM domains adopt similar folds, they are versatile in their binding properties: some SAM domains form homodimers or polymers; others can bind to other SAM domains, to nonSAM domain-containing proteins and even to RNA (reviewed in [24]). The number of SAM-containing proteins per organism increases with complexity: yeast has 4 , Drosophila 42 and human 206. P. falciparum has four (as determined by text scanning of PlasmoDB), two of which, PfTKL1 and PfTKL3, belong to the TKL group, while the other two (PFI1275w and PF11_0079) possess a kinaselike domain lacking residues that are critical for kinase activity (whether or not these proteins are active kinases 


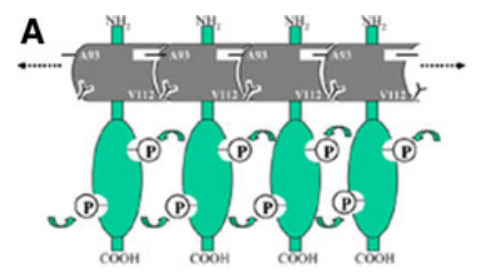

B

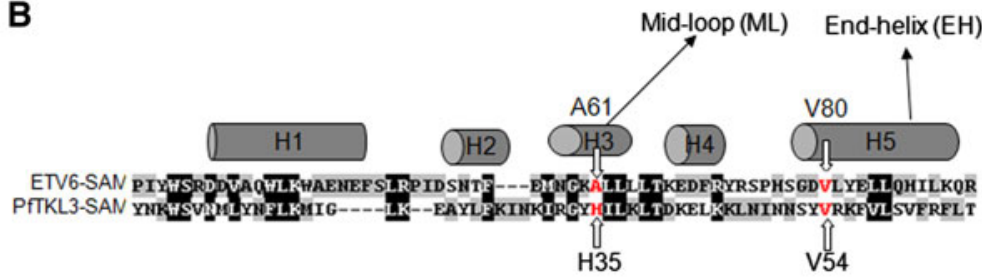

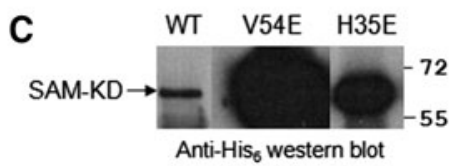

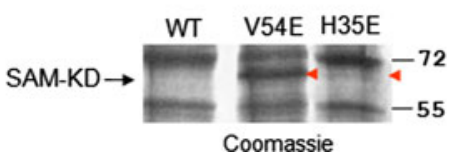

Fig. 8 Mutagenesis of the PfTKL3 SAM domain. a Model for the stimulation of kinase activity by an adjacent SAM domain. Adapted from [23]. b Alignment of the SAM domains of ETV6 and PfTKL3. The five helices in the secondary structure of ETV6 SAM domain are shown as grey cylinders. The ETV6 residues and corresponding PfTKL3 that occur in the interacting interfaces called mid-loop (ML) $\mathrm{H} 3$ and end-helix (EH) $\mathrm{H} 5$ are shown in red. Numbering is from the

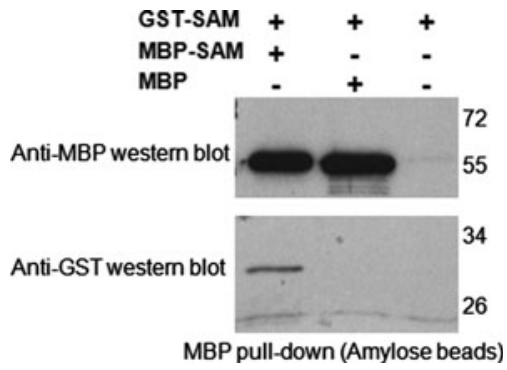

Fig. 9 MBP-SAM domain pull down assay. Purified GST-SAM was incubated with MBP-SAM (lane 1) or MBP alone (lane 2). GSTSAM was incubated with the lysis buffer as negative control (lane 3). Amylose-agarose beads were added to the mixture, incubated and washed. The bound proteins were eluted and subjected to SDS-PAGE and Western blot analysis. The reciprocal experiment (GST pulldown assay) and a gel showing the purified proteins used in this experiment can be found in Supplemental Fig. S4

remains to be established). The two SAM-containing TKLs, in addition to their similarity in gene architecture, also have similar expression profiles (micro-array data available on PlasmoDB) and may possibly interact with each other via their SAM domain. Pfg27/25 is a gametocyte-specific phosphoprotein that can specifically bind single-stranded RNA [25], a property described for some SAM domains [26]. Despite a sequence that is distant from that of typical SAM domains, the Pfg27/25 3D structure displays SAM domain-like folding and was shown to undergo phosphorylation-regulated oligomerisation [27]. This suggests Pfg27/25 is an additional P. falciparum SAM
N-terminal boundary of each SAM domain. c Effect of SAM domain mutations on the recovery of soluble recombinant SAM-KD PfTKL3. Top panel: Western blot (anti-His ${ }_{6}$ ) of wild-type, V54E and H35E recovered from $400 \mathrm{ml}$ of bacterial cultures. Bottom panel: The V54E mutation results in a yield that is sufficient for detection by Coomassie staining

domain-containing protein that may potentially interact with the kinase-related SAM-domain containing proteins.

Interestingly, all four $P$. falciparum SAM-domaincontaining protein kinase-like proteins also carry putative MORN motifs, a feature that, to our knowledge, is not shared by SAM-containing protein kinases from other eukaryotes. MORN motifs may be responsible for localisation at the plasma membrane through direct association with phospholipids [28]. A Toxoplasma gondii protein that comprises repeated MORN motifs associates with structures that form the basal complex, the spindle pole and the apical complex, indicating that MORN motifs, through interaction with lipids, may be the linking membranous region to the cytoskeleton [29-31]. In addition, several MORN motif-containing proteins have been linked to a role in spermatozoa morphogenesis/biogenesis [32, 33]. A role of P. falciparum MORN motifcontaining proteins in the regulation of the cytoskeleton is a definite possibility.

The yeast SAM domain-containing Ste11 MAP3K is essential for mating type differentiation. In Plasmodium, the switch from asexual replication to gametocytogenesis, similar to yeast, is induced by stress factors like starvation, but the signalling pathways involved remain to be identified. Since PfTKL3 is essential for the asexual stage, it is not possible to investigate its possible role in this switching process using the reverse genetics approach we have used here. Approaches such as conditional knock out, promoter swap or conditional protein abundance regulation using a 
destabilisation domain [34] will contribute to the clarification of the possible role of this protein in sexual development.

\section{Subcellular localisation}

In the gametocyte, especially in stage III, the protein accumulates in areas that form thread-like structures that co-localise (or at least closely associate) with $\alpha$-tubulin, most likely at the cytoskeleton micro-tubules. The subpellicular membrane complex is said to elongate and become more prominent in stage II and III gametocytes, and to localise to one side of the cell only, which is also the case for PfTKL3. We speculate that PfTKL3 may be associated with the sub-pellicular membrane complex, thought to have a role in linking the cytoskeleton microtubules to the pellicle membrane in the gametocytes [21]. This is consistent with the presence of a MORN motif on PfTKL3: MORN motifs interact with membrane lipids [28] and have been hypothesised to anchor the cytoskeleton to membranous structures in the apicomplexan T. gondii [30]. The SAM domains of the transcription factor ETV6, of DAG-kinase, of Shank3 and of yeast Ste11 have all been shown to regulate the spatial distribution of their respective proteins [35-38]. The role of the SAM and MORN domains in the localisation of PfTKL3 and other P. falciparum SAM/MORN-containing proteins warrants further studies.

\section{Regulation of PfTKL3 enzymatic activity}

The activity of recombinant PfTKL3 is dramatically stimulated by the presence of the accessory SAM domain, in line with the activation of a PK following translocation of a SAM domain at its N-terminus (reviewed in [23]). Our site-directed mutagenesis and pull-down data suggest that the PfTKL3 SAM domain mediates self-interaction. Polymer formation may explain the reduced yield of soluble recombinant protein and increased activity observed upon addition of the SAM domain. We propose that the PFTKL3 SAM domain, like its analogue in the ETV6-NTRK3 chimeric kinase [23] (see Fig. 8a), mediates oligomerisation of the enzyme and thereby generates a complex where (1) local concentration is very high and (2) the kinase domains are oriented in a way that facilitates trans-autophosphorylation. This is fully consistent with our observations that PfTKL3 autophosphorylation is intermolecular (Fig. 7e).

PfTKL3 is a multi-domain protein with the accessory domains/motifs (14-3-3 mode II, MORN and SAM) that are potentially implicated in various interactions with lipid, protein and even RNA. Phosphorylated PfTKL3 residues, in addition to their role in the regulation of enzymatic activity, may act as docking pads or anchors for other proteins, as established for several (especially membranebound) PKs in other contexts (see Refs. [39, 40], for examples). PfTKL3 may thus act as a hub for a network of interactions, and it will undoubtedly be fascinating to identify interacting partners. The disruption of proteinprotein interactions (PPI) is now considered as a target for chemotherapy [41, 42]. With respect to PfTKL3, which we validated by reverse genetics as a potential target, the inhibition of SAM-mediated interactions offers another opportunity for intervention (in addition to the classical approach targeting catalytic activity). In the past PPI was considered to be un-druggable by small molecule inhibitors because of the often large and featureless interaction interfaces that lack crevices and pockets. However, in recent years substantial progress has been made in the discovery of potent and selective inhibitors of proteinprotein interactions, and such agents are currently in phase II clinical trial for the treatment of several cancers (reviewed in [42, 43].

In summary, the facts that PfTKL3 (1) is a functionally validated target and (2) can be expressed as a strongly active recombinant protein if the SAM domain is included justifies earmarking this protein for high-throughput screening against chemical libraries to identify inhibitory hits compounds as the first step in a drug discovery program targeting this enzyme.

Acknowledgments This work is based on gene identification made possible by the availability of the genome sequences of $P$. falciparum accessible on the PlasmoDB database, an immensely useful tool in malaria research. Financial support for the Plasmodium Genome Consortium was provided by the Burroughs Wellcome Fund, the Wellcome Trust, the National Institutes of Health (NIAID) and the US Department of Defense, Military Infectious Diseases Research Program. Financial Support for PlasmoDB was provided by the Burroughs Wellcome Fund. Work in the C.D. Laboratory is supported by Inserm, the FP6 (SIGMAL and ANTIMAL projects, BioMalPar Network of Excellence) and FP7 (MALSIG project and EviMalar network of Excellence) programmes of the European Commission. AA benefits from a $\mathrm{PhD}$ studentship from the international $\mathrm{PhD}$ programme of the FP6 ANTIMAL Integrated Project (contract LSHPCT-2005-0188).

Open Access This article is distributed under the terms of the Creative Commons Attribution Noncommercial License which permits any noncommercial use, distribution, and reproduction in any medium, provided the original author(s) and source are credited.

\section{References}

1. White NJ, Nosten F, Looareesuwan S, Watkins WM, Marsh K, Snow RW, Kokwaro G, Ouma J, Hien TT, Molyneux ME, Taylor TE, Newbold CI, Ruebush TK 2nd, Danis M, Greenwood BM, Anderson RM, Olliaro P (1999) Averting a malaria disaster. Lancet 353:1965-1967 
2. Snow RW, Trape JF, Marsh K (2001) The past, present and future of childhood malaria mortality in Africa. Trends Parasitol 17:593-597

3. Dondorp AM, Nosten F, Yi P, Das D, Phyo AP, Tarning J, Lwin KM, Ariey F, Hanpithakpong W, Lee SJ, Ringwald P, Silamut K, Imwong M, Chotivanich K, Lim P, Herdman T, An SS, Yeung S, Singhasivanon P, Day NP, Lindegardh N, Socheat D, White NJ (2009) Artemisinin resistance in plasmodium falciparum malaria. N Engl J Med 361:455-467

4. Dondorp AM, Yeung S, White L, Nguon C, Day NP, Socheat D, von Seidlein L. Artemisinin resistance: current status and scenarios for containment. Nat Rev Microbiol 8:272-280

5. Hanks SK (2003) Genomic analysis of the eukaryotic protein kinase superfamily: a perspective. Genome Biol 4:111

6. Zhang J, Yang PL, Gray NS (2009) Targeting cancer with small molecule kinase inhibitors. Nat Rev Cancer 9:28-39

7. Schreiber M, Res I, Matter A (2009) Protein kinases as antibacterial targets. Curr Opin Cell Biol 21:325-330

8. Doerig C, Billker O, Haystead T, Sharma P, Tobin AB, Waters NC (2008) Protein kinases of malaria parasites: an update. Trends Parasitol 24:570-577

9. Doerig C, Meijer L (2007) Antimalarial drug discovery: targeting protein kinases. Expert Opin Ther Targets 11:279-290

10. Ward P, Equinet L, Packer J, Doerig C (2004) Protein kinases of the human malaria parasite Plasmodium falciparum: the kinome of a divergent eukaryote. BMC Genomics 5:79

11. Singh M, Mukherjee P, Narayanasamy K, Arora R, Sen SD, Gupta S, Natarajan K, Malhotra P (2009) Proteome analysis of Plasmodium falciparum extracellular secretory antigens at asexual blood stages reveals a cohort of proteins with possible roles in immune modulation and signaling. Mol Cell Proteomics 8:21022118

12. Tamura K, Dudley J, Nei M, Kumar S (2007) MEGA4: molecular evolutionary genetics analysis (MEGA) software version 4.0. Mol Biol Evol 24:1596-1599

13. Dorin D, Alano P, Boccaccio I, Ciceron L, Doerig C, Sulpice R, Parzy D, Doerig C (1999) An atypical mitogen-activated protein kinase (MAPK) homologue expressed in gametocytes of the human malaria parasite Plasmodium falciparum. Identification of a MAPK signature. J Biol Chem 274:29912-29920

14. Carter R, Ranford-Cartwright L, Alano P (1993) The culture and preparation of gametocytes of Plasmodium falciparum for immunochemical, molecular, and mosquito infectivity studies. Methods Mol Biol 21:67-88

15. Ho SN, Hunt HD, Horton RM, Pullen JK, Pease LR (1989) Sitedirected mutagenesis by overlap extension using the polymerase chain reaction. Gene 77:51-59

16. Sidhu AB, Valderramos SG, Fidock DA (2005) pfmdr1 mutations contribute to quinine resistance and enhance mefloquine and artemisinin sensitivity in Plasmodium falciparum. Mol Microbiol 57:913-926

17. Dorin-Semblat D, Sicard A, Doerig C, Ranford-Cartwright L (2008) Disruption of the PfPK7 gene impairs schizogony and sporogony in the human malaria parasite Plasmodium falciparum. Eukaryot Cell 7:279-285

18. Fennell C, Babbitt S, Russo I, Wilkes J, Ranford-Cartwright L, Goldberg DE, Doerig C (2009) PfeIK1, a eukaryotic initiation factor 2alpha kinase of the human malaria parasite Plasmodium falciparum, regulates stress-response to amino-acid starvation. Malar J 8:99

19. Reininger L, Tewari R, Fennell C, Holland Z, Goldring D, Ranford-Cartwright L, Billker O, Doerig C (2009) An essential role for the plasmodium Nek-2 nima-related protein kinase in the sexual development of malaria parasites. J Biol Chem 284: 20858-20868
20. Le Roch KG, Zhou Y, Blair PL, Grainger M, Moch JK, Haynes JD, De La Vega P, Holder AA, Batalov S, Carucci DJ, Winzeler EA (2003) Discovery of gene function by expression profiling of the malaria parasite life cycle. Science 301:1503-1508

21. Sinden RE (1982) Gametocytogenesis of Plasmodium falciparum in vitro: an electron microscopic study. Parasitology 84:1-11

22. Tognon CE, Mackereth CD, Somasiri AM, McIntosh LP, Sorensen PH (2004) Mutations in the SAM domain of the ETV6NTRK3 chimeric tyrosine kinase block polymerization and transformation activity. Mol Cell Biol 24:4636-4650

23. Lannon CL, Sorensen PH (2005) ETV6-NTRK3: a chimeric protein tyrosine kinase with transformation activity in multiple cell lineages. Semin Cancer Biol 15:215-223

24. Qiao F, Bowie JU (2005) The many faces of SAM. Sci STKE 2005: re7

25. Sharma A, Sharma I, Kogkasuriyachai D, Kumar N (2003) Structure of a gametocyte protein essential for sexual development in Plasmodium falciparum. Nat Struct Biol 10:197-203

26. Aviv T, Lin Z, Lau S, Rendl LM, Sicheri F, Smibert CA (2003) The RNA-binding SAM domain of Smaug defines a new family of post-transcriptional regulators. Nat Struct Biol 10:614-621

27. Camarda G, Bertuccini L, Singh SK, Salzano AM, Lanfrancotti A, Olivieri A, Scaloni A, Sharma A, Alano P (2009) Regulated oligomerisation and molecular interactions of the early gametocyte protein Pfg27 in Plasmodium falciparum sexual differentiation. Int J Parasitol 40:663-673

28. Ma H, Lou Y, Lin WH, Xue HW (2006) MORN motifs in plant PIPKs are involved in the regulation of subcellular localization and phospholipid binding. Cell Res 16:466-478

29. Ferguson DJ, Sahoo N, Pinches RA, Bumstead JM, Tomley FM, Gubbels MJ (2008) MORN1 has a conserved role in asexual and sexual development across the apicomplexa. Eukaryot Cell 7:698-711

30. Gubbels MJ, Vaishnava S, Boot N, Dubremetz JF, Striepen B (2006) A MORN-repeat protein is a dynamic component of the Toxoplasma gondii cell division apparatus. J Cell Sci 119:22362245

31. Hu K (2008) Organizational changes of the daughter basal complex during the parasite replication of Toxoplasma gondii. PLoS Pathog 4:e10

32. Choi YJ, Hwang KC, Park JY, Park KK, Kim JH, Park SB, Hwang S, Park H, Park C (2010) Identification and characterization of a novel mouse and human MOPT gene containing MORN-motif protein in testis. Theriogenology 73:273-281

33. Tokuhiro $K$, Hirose $M$, Miyagawa $Y$, Tsujimura A, Irie $S$, Isotani A, Okabe M, Toyama Y, Ito C, Toshimori K, Takeda K, Oshio S, Tainaka H, Tsuchida J, Okuyama A, Nishimune Y, Tanaka H (2008) Meichroacidin containing the membrane occupation and recognition nexus motif is essential for spermatozoa morphogenesis. J Biol Chem 283:19039-19048

34. Armstrong CM, Goldberg DE (2007) An FKBP destabilization domain modulates protein levels in Plasmodium falciparum. Nat Methods 4:1007-1009

35. Baron MK, Boeckers TM, Vaida B, Faham S, Gingery M, Sawaya MR, Salyer D, Gundelfinger ED, Bowie JU (2006) An architectural framework that may lie at the core of the postsynaptic density. Science 311:531-535

36. Harada BT, Knight MJ, Imai S, Qiao F, Ramachander R, Sawaya MR, Gingery M, Sakane F, Bowie JU (2008) Regulation of enzyme localization by polymerization: polymer formation by the SAM domain of diacylglycerol kinase delta1. Structure 16:380-387

37. Kim CA, Phillips ML, Kim W, Gingery M, Tran HH, Robinson MA, Faham S, Bowie JU (2001) Polymerization of the SAM domain of TEL in leukemogenesis and transcriptional repression. EMBO J 20:4173-4182 
38. Wu C, Jansen G, Zhang J, Thomas DY, Whiteway M (2006) Adaptor protein Ste50p links the Ste11p MEKK to the HOG pathway through plasma membrane association. Genes Dev 20:734-746

39. Ingley E (2008) Src family kinases: regulation of their activities, levels and identification of new pathways. Biochim Biophys Acta 1784:56-65

40. Tzivion G, Shen YH, Zhu J (2001) 14-3-3 proteins; bringing new definitions to scaffolding. Oncogene 20:6331-6338
41. Wells JA, McClendon CL (2007) Reaching for high-hanging fruit in drug discovery at protein-protein interfaces. Nature 450:10011009

42. Arkin M (2005) Protein-protein interactions and cancer: small molecules going in for the kill. Curr Opin Chem Biol 9:317-324

43. van Montfort RL, Workman P (2009) Structure-based design of molecular cancer therapeutics. Trends Biotechnol 27:315-328 